\title{
1
}

\section{Introduction to Satellite Communications}

A communications satellite is an orbiting artificial earth satellite that receives a communications signal from a transmitting ground station, amplifies and possibly processes it, then transmits it back to the earth for reception by one or more receiving ground stations. Communications information neither originates nor terminates at the satellite itself. The satellite is an active transmission relay, similar in function to relay towers used in terrestrial microwave communications.

The commercial satellite communications industry has its beginnings in the mid-1960s, and in less than 50 years has progressed from an alternative exotic technology to a mainstream transmission technology, which is pervasive in all elements of the global telecommunications infrastructure. Today's communications satellites offer extensive capabilities in applications involving data, voice, and video, with services provided to fixed, broadcast, mobile, personal communications, and private networks users.

Satellite communications are now an accepted fact of everyday life, as evidenced by the antennas or 'dishes' that dot city and country horizons, or the nearly instantaneous global news coverage that is taken for granted, particularly in times of international crises.

The communications satellite is a critical element in the overall telecommunications infrastructure, as represented by Figure 1.1, which highlights, by the shaded area, the communications satellite component as related to the transmission of information. Electronic information in the form of voice, data, video, imaging, etc., is generated in a user environment on or near the earth's surface. The information's first node is often a terrestrial interface, which then directs the information to a satellite uplink, which generates an RF (radio frequency) radiowave that propagates through the air link to an orbiting satellite (or satellites). The information bearing radiowave is amplified and possibly processed at the satellite, then reformatted and transmitted back to a receiving ground station through a second RF radiowave propagating through the air link. Mobile users, indicated by the vehicle and handheld phone on the figure, generally bypass the terrestrial interface only for direct mobile-to-mobile communications.

Satellite Communications Systems Engineering Louis J. Ippolito, Jr.

(C) 2008 John Wiley \& Sons, Ltd 


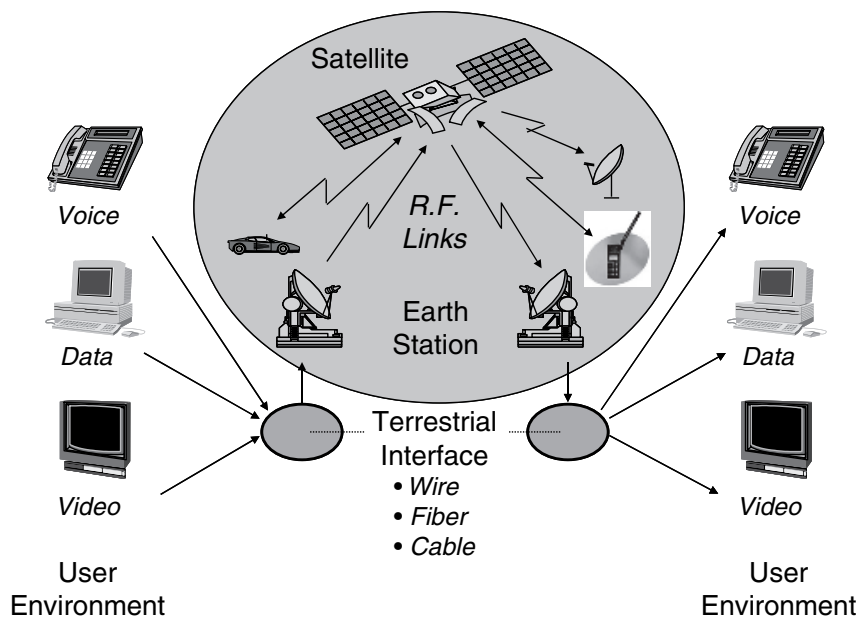

Figure 1.1 Communications via satellite in the telecommunications infrastructure

Communications by satellite offers a number of features that are not readily available with alternative modes of transmission, such as terrestrial microwave, cable, or fiber networks. Some of the advantages of satellite communications are:

- Distance Independent Costs. The cost of satellite transmission is basically the same, regardless of the distance between the transmitting and receiving earth stations. Satellite based transmission costs tend to be more stable, particularly for international or intercontinental communications over vast distances.

- Fixed Broadcast Costs. The cost of satellite broadcast transmission, that is, transmission from one transmit ground terminal to a number of receiving ground terminals, is independent of the number of ground terminals receiving the transmission.

- High Capacity. Satellite communications links involve high carrier frequencies, with large information bandwidths. Capacities of typical communications satellites range from 10s to 100s of Mbps (Mega-bits per second), and can provide services for several hundred video channels or several tens of thousands of voice or data links.

- Low Error Rates. Bit errors on a digital satellite link tend to be random, allowing statistical detection and error correction techniques to be used. Error rates of one bit error in $10^{6}$ bits or better can be routinely achieved efficiently and reliably with standard equipment.

- Diverse User Networks. Large areas of the earth are visible from the typical communications satellite, allowing the satellite to link together many users simultaneously. Satellites are particularly useful for accessing remote areas or communities not otherwise accessible by terrestrial means. Satellite terminals can be on the surface, at sea, or in the air, and can be fixed or mobile.

The successful implementation of satellite wireless communications requires robust air links providing the uplink and downlink paths for the communications signal. Transmission through the atmosphere will degrade signal characteristics however, and under some conditions it can 
be the major impediment to successful system performance. A detailed knowledge of the types of atmospheric effects that impact satellite communications and the means to predict and model them for application to communications link design and performance is essential for wireless satellite link engineering. The effects of the atmosphere are even more significant as current and planned satellites move up to higher operating frequencies, including the $\mathrm{Ku}$-band $(14 \mathrm{GHz}$ uplink/12 GHz downlink), Ka-band $(30 \mathrm{GHz} / 20 \mathrm{GHz})$, and V-band $(50 \mathrm{GHz} / 40 \mathrm{GHz})$, where the effects of rain, gaseous attenuation, and other effects will increase.

\subsection{Early History of Satellite Communications}

The idea of a synchronous orbiting satellite capable of relaying communications to and from the earth is generally attributed to Arthur C. Clarke. Clarke observed in his classic 1945 paper [1] that a satellite in a circular equatorial orbit with a radius of about $36000 \mathrm{~km}$ would have an angular velocity matching that of the earth, and thus it would remain above the same spot on the earth's surface. This orbiting artificial satellite could therefore receive and transmit signals from anywhere on earth in view of the satellite to any other place on the surface in view of the satellite.

The technology to verify this concept was not available until over a decade later, with the launch in 1957 of SPUTNIK I by the former USSR. This launch ushered in the 'space age' and both the United States and the USSR began robust space programs to develop the technology and to apply it to emerging applications. A brief summary of some of the early communications satellites programs, and their major accomplishments, follows.

\section{SCORE}

The first communications by artificial satellite was accomplished by SCORE (Signal Communicating by Orbiting Relay Equipment), launched by the Air Force into a low (160 by $1280 \mathrm{~km}$ ) orbit in December 1958. SCORE relayed a recorded voice message, on a delayed basis, from one earth station to another. SCORE broadcast a message from President Eisenhower to stations around the world, giving the first hint of the impact that satellites would have on point-to-point communications. The maximum message length was four minutes, and the relay operated on a $150 \mathrm{MHz}$ uplink and $108 \mathrm{MHz}$ downlink. SCORE, powered by battery only, operated for 12 days before its battery failed, and decayed out of orbit 22 days later [2].

\section{ECHO}

The first of several efforts to evaluate communications relay by passive techniques was initiated with the ECHO satellites 1 and 2, launched by the National Aeronautics and Space Administration (NASA) in August 1960 and January 1964, respectively. The ECHO satellites were large orbiting spheres of aluminized Mylar, over $30 \mathrm{~m}$ in diameter, which served as passive reflectors for signals transmitted from stations on the earth. They caught the interest of the public because they were visible from the earth with the unaided eye under the right lighting conditions, usually just as the sun was rising or setting. The ECHO relays operated at frequencies from 162 to $2390 \mathrm{MHz}$, and required large ground terminal antennas, typically $18 \mathrm{~m}$ or more, with transmit powers of $10 \mathrm{~kW}$. ECHO 1 remained in orbit for nearly 8 years, and ECHO 2 for over 5 years [3]. 


\section{COURIER}

Launched in October 1960, COURIER extended SCORE delayed repeater technology and investigated store-and-forward and real-time capabilities from a low orbiting satellite. COURIER operated with an uplink frequency of 1.8 to $1.9 \mathrm{GHz}$, and a downlink of 1.7 to $1.8 \mathrm{GHz}$. It was all solid state except for the 2-watt output power tubes, and was the first artificial satellite to employ solar cells for power. The satellite performed successfully for 17 days, until a command system failure ended operations [4].

\section{WESTFORD}

WESTFORD was the second technology employed to evaluate communications relay by passive techniques, with a first successful launch by the US Army in May 1963. WESTFORD consisted of tiny resonant copper dipoles dispersed in an orbital belt, with communications accomplished by reflection from the dispersed dipole reflectors. The dipoles were sized to the half wavelength of the relay frequency, $8350 \mathrm{MHz}$. Voice and frequency shift keyed (FSK) transmissions up to $20 \mathrm{kbps}$ were successfully transmitted from a ground station in California to one in Massachusetts. As the belt dispersed, however, the link capacity dropped to below $100 \mathrm{bps}$. The rapid development of active satellites reduced interest in passive communications, and ECHO and WESTFORD brought passive technology experiments to an end [5].

\section{TELSTAR}

The TELSTAR Satellites 1 and 2, launched into low orbits by NASA for AT\&T/Bell Telephone Laboratories in July 1962 and May 1963, respectively, were the first active wideband communications satellites. TELSTAR relayed analog FM signals, with a $50 \mathrm{MHz}$ bandwidth, and operated at frequencies of $6.4 \mathrm{GHz}$ on the uplink and $4.2 \mathrm{GHz}$ on the downlink. These frequencies led the way for $6 / 4 \mathrm{GHz} \mathrm{C}$-band operation, which currently provides the major portion of fixed satellite service (FSS) throughout the world. TELSTAR 1 provided multichannel telephone, telegraph, facsimile, and television transmissions to stations in the United States, Britain, and France until the command subsystem failed in November 1962 due to Van Allen belt radiation. TELSTAR 2, redesigned with radiation resistant transistors and launched into a higher orbit to decrease exposure in the Van Allen belts, operated successfully for two years [6].

\section{RELAY}

RELAY 1, developed by RCA for NASA, was launched in December 1962 and operated for 14 months. RELAY had two redundant repeaters, each with a $25 \mathrm{MHz}$ channel and two $2 \mathrm{MHz}$ channels. It operated with $1725 \mathrm{MHz}$ uplink and $4160 \mathrm{MHz}$ downlink frequencies, and had a 10-watt TWT (traveling wave tube) output amplifier. Extensive telephony and network television transmissions were accomplished between the United States, Europe, and Japan. RELAY 2 was launched in January 1964 and again operated for 14 months. The RELAY and TELSTAR programs demonstrated that reliable, routine communications could be accomplished from orbiting satellites, and further indicated that satellite systems could share frequencies with terrestrial systems without interference degradations [7].

\section{SYNCOM}

The SYNCOM satellites, developed by Hughes Aircraft Company for NASA GSFC, provided the first communications from a synchronous satellite. SYNCOM 2 and 3 were placed in orbit in July 1963 and July 1964, respectively (SYNCOM 1 failed at launch). SYNCOM, with $7.4 \mathrm{GHz}$ uplink and $1.8 \mathrm{GHz}$ downlink frequencies, employed two $500 \mathrm{kHz}$ channels 
for two-way narrowband communications, and one $5 \mathrm{MHz}$ channel for one-way wideband transmission. SYNCOM was the first testbed for the development of station keeping and orbital control principles for synchronous satellites. It was the first satellite to employ range and rangerate tracking. NASA conducted voice, teletype, and facsimile tests, including extensive public demonstrations to increase the base of satellite communications interest. The US Department of Defense also conducted tests using SYNCOM 2 and 3, including transmissions with a shipboard terminal. Tests with aircraft terminals were also conducted with the SYNCOM VHF command and telemetry links [8].

\section{EARLYBIRD}

The first commercial operational synchronous communications satellite was EARLYBIRD, later called INTELSAT I, developed by COMSAT for INTELSAT, and launched by NASA in April 1965. The communications subsystem, very similar to the SYNCOM 3 design, had two $25 \mathrm{MHz}$ transponders and operated at C-band, with uplinks at $6.3 \mathrm{GHz}$ and downlinks at 4.1 GHz. It had a capacity of 240 two-way voice circuits or one two-way television circuit. TWT output power was 6 watts. Operations between the US and Europe began on June 28, 1965 , a date that many recognize as the birth date of commercial satellite communications. EARLYBIRD remained in service until August 1969, when the later generation INTELSAT III satellites replaced it [9].

\section{ATS-1 (Applications Technology Satellite 1)}

The ATS-1, first of NASA's highly successful series of Applications Technology Satellites, was launched in December 1966 and demonstrated a long list of 'firsts' in satellite communications. ATS-1 included an electronically despun antenna with $18-\mathrm{dB}$ gain and a $17^{\circ}$ beamwidth. It operated at C-band (6.3 GHz uplink, $4.1 \mathrm{GHz}$ downlink), with two $25 \mathrm{MHz}$ repeaters. ATS-1 provided the first multiple access communications from synchronous orbit. ATS-1 had VHF links (149 MHz uplink, $136 \mathrm{MHz}$ downlink) for the evaluation of air to ground communications via satellite. ATS-1 also contained a high-resolution camera, providing the first photos of the full earth from orbit. ATS-1 continued successful operation well beyond its three year design life, providing VHF communications to the Pacific basin region until 1985, when station keeping control was lost [7].

\section{ATS-3}

The ATS-3, launched in November 1967, continued experimental operations in the C and VHF bands, with multiple access communications and orbit control techniques. ATS-3 allowed, for the first time, 'cross-strap' operation at C-band and VHF; the signal received at VHF could be transmitted to the ground at C-band. ATS-3 provided the first color high-resolution pictures of the now familiar 'blue marble' earth as seen from synchronous orbit. ATS-3, like ATS-1, far exceeded its design life, providing VHF communications to the Pacific and continental United States for public service applications for over a decade [7].

\section{ATS-5}

ATS-5 had a C-band communications subsystem similar to its predecessors, but did not have the VHF capability. Instead it had an L-band (1650 MHz uplink, $1550 \mathrm{MHz}$ downlink) subsystem to investigate air to ground communications for navigation and air traffic control. ATS-5 also contained a millimeter wave experiment package that operated at $31.65 \mathrm{GHz}$ (uplink) and 15.3 GHz (downlink), designed to provide propagation data on the effects of the atmosphere 
on earth-space communications at these frequencies. ATS-5 was designed to operate as a gravity gradient stabilized satellite, unlike the earlier spin-stabilized ATS-1 and -3 satellites. It was successfully launched in August 1969 into synchronous orbit, but the gravity stabilization boom could not be deployed because of the satellite's spin condition. ATS-5 was placed into a spin-stabilized condition, resulting in the satellite antennas sweeping the earth once every 860 milliseconds. Most of the communications experiments performed with limited success in this unexpected 'pulsed' operation mode. The $15.3 \mathrm{GHz}$ millimeter wave experiment downlink, however, was able to function well, after modifications to the ground terminal receivers, and extensive propagation data were accumulated at over a dozen locations in the United States and Canada [7].

\section{ANIK A}

ANIK A (initially called ANIK I), launched in November 1972 by NASA for Telsat Canada, was the first domestic commercial communications satellite. Two later ANIK As were launched in April 1973 and May 1975. The satellites, built by Hughes Aircraft Company, operated at C-band and had 12 transponders, each $36 \mathrm{MHz}$ wide. The primary services provided were television distribution, SCPC (single channel per carrier) voice, and data services. The transmit power was 5 watts, with a single beam covering most of Canada and the northern United States. The antenna pattern for ANIK A was optimized for Canada, however sufficient coverage of the northern US was available to allow leased service by US communications operators for domestic operations prior to the availability of US satellites. The ANIK A series continued in service until 1985, when ANIK D satellites replaced them [10].

\section{ATS-6}

ATS-6, the second generation of NASA's Applications Technology Satellite program, provided major advancements in communications satellite technology and in new applications demonstrations. ATS-6 consisted of a 9 m diameter deployed parabolic antenna, earth viewing module, two sun-seeking solar arrays, and the supporting structures [11]. It was launched in May 1974 and positioned at $94^{\circ} \mathrm{W}$ longitude, where it remained for one year. In July 1975 it was moved to $35^{\circ} \mathrm{E}$ longitude for instructional television experiments to India. After one year it was again relocated to $140^{\circ} \mathrm{W}$ longitude and used for several experimental programs until it was moved out of synchronous orbit in 1979. ATS-6 had eight communications and propagation experiments that covered a frequency range from $860 \mathrm{MHz}$ to $30 \mathrm{GHz}$. The communications subsystems on ATS-6 included four receivers: $1650 \mathrm{MHz}$ (L-band), $2253 \mathrm{MHz}$ (S-band), 5925-6425 (C-band), and 13/18 GHz (K-band). Transmitter frequencies were: $860 \mathrm{MHz}$ (L-band), $2063 \mathrm{MHz}$ (S-band), 3953-4153 MHz (C-band), and 20/30 GHz ( $\mathrm{K}_{\mathrm{a}}$-band). The ATS-6 provided cross-strapping at Intermediate Frequency (IF) between any receiver to any transmitter (except for the $13 / 18 \mathrm{GHz}$ receiver, which operated with a $4150 \mathrm{MHz}$ transmitter only), allowing a wide range of communications modes. Major experiments [12] were:

- Position Location and Aircraft Communication Experiment (PLACE), which consisted of voice and digital data transmissions and four-tone ranging for aircraft position location. The system allowed multiple access voice from up to 100 aircraft, operating in $10 \mathrm{KHz}$ channels.

- Satellite Instructional Television Experiment (SITE), a cooperative experiment between NASA and the government of India to demonstrate direct broadcast satellite television for 
instructional purposes. Satellite signals at $860 \mathrm{MHz}$ were received at over 2000 villages, using simple $3 \mathrm{~m}$ parabolic antennas.

- Television Relay Using Small Terminals (TRUST), which evaluated hardware and system performance for $860 \mathrm{MHz}$ satellite broadcast television, using the same general configuration as the SITE.

- Health Education Experiment (HEW), which provided satellite distribution of educational and medical programming, primarily to Alaska, the Rocky Mountain states, and Appalachia. Two independent steerable beams, operating through the $9 \mathrm{~m}$ reflector, were available, with the uplink at C-band $(5950 \mathrm{MHz})$ and the downlink at S-band $(2750$ and $2760 \mathrm{MHz})$.

- Radio Frequency Interference Experiment (RFI), which monitored the 5925 to $6425 \mathrm{MHz}$ band with a sensitive on-board receiver to measure and map radio frequency interference sources in the continental United States (CONUS). The minimum detectable source EIRP was $10 \mathrm{dBW}$, with a frequency resolution of $10 \mathrm{kHz}$.

- NASA Millimeter Wave Propagation Experiment, which was designed to provide information on the communications and propagation characteristics of the atmosphere at 20 and $30 \mathrm{GHz}\left(\mathrm{K}_{\mathrm{a}}\right.$-band). Two modes of operation were available: i) downlink beacons at 20 and $30 \mathrm{GHz}$ for measurement of rain attenuation, atmospheric absorption, and other effects; and ii) a communications mode, with a $\mathrm{C}$-band $(6 \mathrm{GHz})$ uplink, and simultaneous downlinks at 20,30 , and $4 \mathrm{GHz}$, for the evaluation of millimeter wave communications in a $40 \mathrm{MHz}$ bandwidth. Extensive measurements were obtained in the US and Europe, providing the first detailed information on $\mathrm{K}_{\mathrm{a}}$-band satellite communications performance.

- COMSAT Millimeter Wave Experiment, which consisted of 39 uplinks, 15 at $13.19 \mathrm{GHz}$, and 24 at $17.79 \mathrm{GHz}$, received by ATS- 6 and retransmitted to the ground at C-band $(4150 \mathrm{MHz})$. About one year of measurements were accumulated on rain attenuation statistics, joint probability distributions, and required rain margins, for links operating at K-band.

The accomplishments of ATS-6 have been extensively documented, and have provided a wide range of valuable design and performance information for virtually every application implemented in current satellite communications systems.

\section{CTS}

The Communications Technology Satellite (CTS) was a joint program between NASA and the Canadian Department of Communications, to evaluate high power satellite technology applicable to broadcast satellite service (BSS) applications at $\mathrm{K}_{\mathrm{u}}$-band. A $12 \mathrm{GHz}, 200$-watt output TWT on CTS, provided by NASA, allowed reception of television and two-way voice with small $(120 \mathrm{~cm}$ diameter) ground terminal antennas [13]. A continuously operating $11.7 \mathrm{GHz}$ propagation beacon was also included, and long-term (36 month) propagation statistics were developed for several locations in the United States [14]. CTS was launched in January 1976, and provided extensive experimental tests and demonstrations in the US and Canada until operations ended in November 1979.

Three important events helped to shape the direction and speed of satellite communications development in its early years:

- United Nations Initiative of 1961 - This initiative stated that 'communications by means of satellite should be available to the nations of the world as soon as practicable...'. 
- COMSAT Act of 1962 - The Congress of the United States created an international communications satellite organization, COMSAT. COMSAT was incorporated in 1963 and served as the primary commercial provider of international satellite communications services in the United States.

- INTELSAT - In August 1964, INTELSAT was created, becoming the recognized international legal entity for international satellite communications. COMSAT is the sole United States conduit organization to INTELSAT.

These early accomplishments and events led to the rapid growth of the satellite communications industry beginning in the mid-1960s. INTELSAT was the prime mover in this time period, focusing on the first introduction of the benefits of satellite communications to many nations across the globe.

The decade of the 1970s saw the advent of domestic satellite communications (i.e., the provision of satellite services within the domestic boundaries of a single country), led by the rapid reduction in the cost of satellite equipment and services. The technology of the 1970s also allowed the first consideration of regional satellite communications, with antennal coverage areas over several contiguous countries with similar communications interests.

The 1980s began the rapid introduction of new satellite services and new participants in satellite communications. Nearly 100 countries were involved in satellite communications providing either satellite systems or satellite-based services. This decade also saw the advent of new and innovative ways to pay for the high costs of satellite systems and services, including lease/buy options, private networks (often referred to as very small antenna terminals or VSATs), and private launch services.

The 1990s introduced mobile and personal communications services via satellite. This era also saw the move to higher RF frequencies to support the increasing data rate requirements in the midst of bandwidth saturation in the lower allocated frequency bands. 'Smart satellites' were also introduced, providing on-board processing and other advanced techniques on the satellite itself, morphing the satellite from a mere data relay to a major communications processing hub in the sky.

The new millennium has seen the rapid introduction of new services, including direct to the home video and audio broadcasting and cellular mobile satellite communications networks. The preferred orbit for communications satellites, the geosynchronous (GSO) orbit, now shared the spotlight with low orbit non-GSO (NGSO) networks, particularly for global cellular mobile communications.

Since its inception, the satellite communications industry has been characterized by a vigorous expansion to new markets and applications, which exploit the advantages of the satellite link and provide cost effective alternatives to the traditional modes of telecommunications transmission.

\subsection{Some Basic Communications Satellite System Definitions}

This section provides some of the basic definitions and parameters used in the satellite communications industry, which will be used throughout the book in the evaluation and analysis of satellite communications systems design and performance. The relevant sections that discuss the parameters more fully are also indicated where appropriate. 


\subsubsection{Satellite Communications Segments}

We begin with the communications satellite portion of the communications infrastructure, shown by the shaded oval in Figure 1.1. The satellite communications portion is broken down into two areas or segments: the space segment and the ground (or earth) segment.

\subsubsection{Space Segment}

The elements of the space segment of a communications satellite system are shown on Figure 1.2. The space segment includes the satellite (or satellites) in orbit in the system, and the ground station that provides the operational control of the satellite(s) in orbit. The ground station is variously referred to as the Tracking, Telemetry, Command $(T T \& C)$ or the Tracking, Telemetry, Command and Monitoring (TTC\&M) station. The TTC\&M station provides essential spacecraft management and control functions to keep the satellite operating safely in orbit. The TTC\&M links between the spacecraft and the ground are usually separate from the user communications links. TTC\&M links may operate in the same frequency bands or in other bands. TTC\&M is most often accomplished through a separate earth terminal facility specifically designed for the complex operations required to maintain a spacecraft in orbit. The TTC\&M functions and subsystems are described in more detail in Chapter 3, Section 3.1.6.

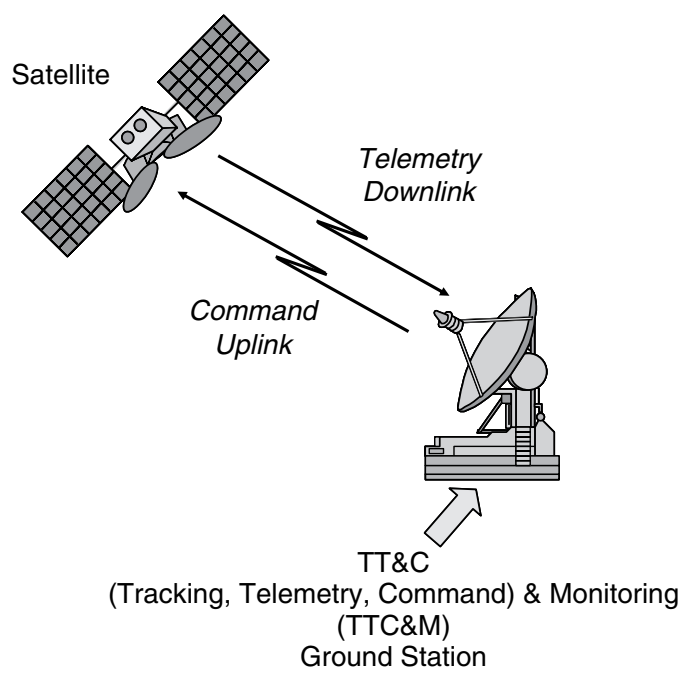

Figure 1.2 The space segment for a communications satellite network

\subsubsection{Ground Segment}

The ground segment of the communications satellite system consists of the earth surface area based terminals that utilize the communications capabilities of the Space Segment. TTC\&M 
ground stations are not included in the ground segment. The ground segment terminals consist of three basic types:

- fixed (in-place) terminals;

- transportable terminals;

- mobile terminals.

Fixed terminals are designed to access the satellite while fixed in-place on the ground. They may be providing different types of services, but they are defined by the fact that they are not moving while communicating with the satellite. Examples of fixed terminals are small terminals used in private networks (VSATs), or terminals mounted on residence buildings used to receive broadcast satellite signals.

Transportable terminals are designed to be movable, but once on location remain fixed during transmissions to the satellite. Examples of the transportable terminal are satellite news gathering (SGN) trucks, which move to locations, stop in place, and then deploy an antenna to establish links to the satellite.

Mobile terminals are designed to communicate with the satellite while in motion. They are further defined as land mobile, aeronautical mobile, or maritime mobile, depending on their locations on or near the earth surface. Mobile satellite communications are discussed in detail in Chapter 11.

\subsubsection{Satellite Link Parameters}

The communications satellite link is defined by several basic parameters, some used in traditional communications system definitions, others unique to the satellite environment. Figure 1.3 summarizes the parameters used in the evaluation of satellite communications links. Two oneway free-space or air links between Earth Stations $A$ and $B$ are shown. The portion of the link from the earth station to the satellite is called the uplink, while the portion from the satellite to

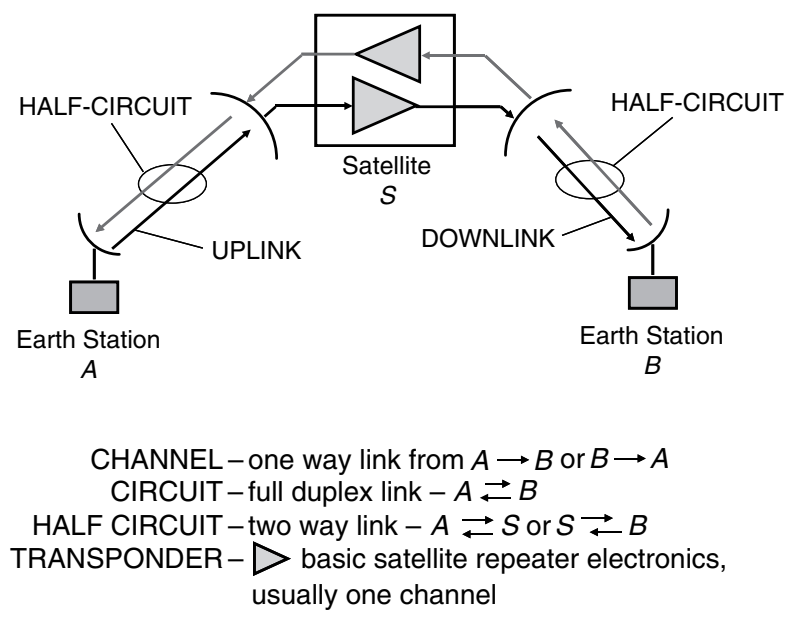

Figure 1.3 Basic link parameters in the communications satellite link 
the ground is the downlink. Note that either station has an uplink and a downlink. The electronics in the satellite that receives the uplink signal, amplifies and possibly processes the signal, and then reformats and transmits the signal back to the ground, is called the transponder, designated by the triangular amplifier symbol in the figure (the point of the triangle indicates the direction of signal transmission). Two transponders are required in the satellite for each twoway link between the two ground stations as shown. The antennas on the satellite that receive and transmit the signals are usually not included as a part of the transponder electronics - they are defined as a separate element of the satellite payload (See Chapter 3).

A channel is defined as the one-way total link from $A$-to- $S$-to- $B$, OR the link from $B$-to- $S$-to- $A$. The duplex (two-way) links $A$-to- $S$-to- $B$ AND $B$-to- $S$-to- $A$ establish a circuit between the two stations. A half-circuit is defined as the two links at one of the earth stations, that is $A$-to- $S$ AND $S$-to- $A$; OR $B$-to- $S$ AND $S$-to- $B$. The circuit designations are a carry-over from standard telephony definitions, which are applied to the satellite segment of the communications infrastructure.

\subsubsection{Satellite Orbits}

The characteristics of satellite orbits in common use for a vast array of satellite communications services and applications are discussed in detail in Chapter 2. We introduce here the satellite orbit terms for the four most commonly used orbits in satellite communications, shown in Figure 1.4. The basic orbit altitude(s) and the one-way delay times are shown for each orbit, along with the common abbreviation designations.
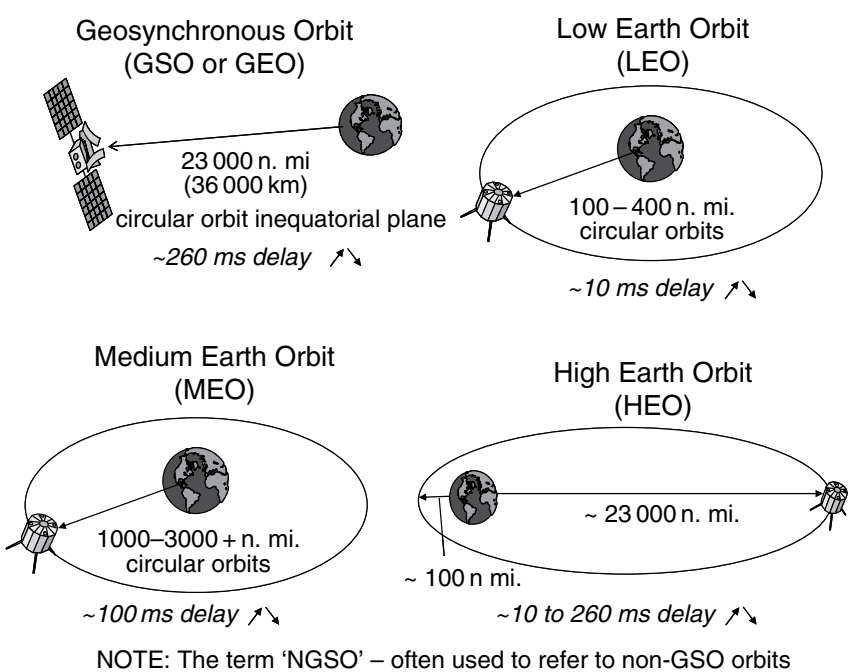

NOTE: The term 'NGSO' - often used to refer to non-GSO orbits

Figure 1.4 Satellite orbits

\subsubsection{Geosynchronous Orbit (GSO or GEO)}

The GSO orbit is by far the most popular orbit used for communications satellites. A GSO satellite is located in a circular orbit in the equatorial plane, at a nominal distance of $36000 \mathrm{~km}$ 
at a stable point, which maintains the satellite at a fixed location in the sky. This is a tremendous advantage for satellite communications, because the pointing direction remains fixed in space and the ground antenna does not need to track a moving satellite. A disadvantage of the GSO is the long delay time of $\sim 260 \mathrm{~ms}$, which can affect network synchronization or impact voice communications. The GSO is described in detail in Chapter 2, Section 2.3.1.

\subsubsection{Low Earth Orbit (LEO)}

The second most common orbit is the low earth orbit (LEO), which is a circular orbit nominally 160 to $640 \mathrm{~km}$ above the earth. The delay is low, $\sim 10 \mathrm{~ms}$, however the satellite moves across the sky, and the ground station must actively track the satellite to maintain communications. The LEO is described in Chapter 2, Section 2.3.2.

\subsubsection{Medium Earth Orbit (MEO)}

The MEO is similar to the LEO, however the satellite is in a higher circular orbit - 1600 to $4200 \mathrm{~km}$. It is a popular orbit for navigation satellites such as the GPS constellation. The MEO is described in Chapter 2, Section 2.3.3.

\subsubsection{High Earth Orbit (HEO)}

The HEO is the only non-circular orbit of the four types. It operates with an elliptical orbit, with a maximum altitude (apogee) similar to the GSO, and a minimum altitude (perigee) similar to the LEO. The HEO, used for special applications where coverage of high latitude locations is required, is discussed in Chapter 2, Section 2.3.4.

Satellite orbits that are not synchronous, such as the LEO, MEO, or HEO, are often referred to as non-geosynchronous orbit (NGSO) satellites.

\subsubsection{Frequency Band Designations}

The frequency of operation is perhaps the major determining factor in the design and performance of a satellite communications link. The wavelength of the free space path signal is the principal parameter that determines the interaction effects of the atmosphere, and the resulting link path degradations. Also, the satellite systems designer must operate within the constraints of international and domestic regulations related to choice of operating free space path frequency.

Two different methods of designation have come into common use to define radio frequency bands. Letter band designations, derived from radar applications in the 1940s, divide the spectrum from 1 to $300 \mathrm{GHz}$ into eight bands with nominal frequency ranges, as shown on Figure 1.5. The $\mathrm{K}$-band is further broken down into $\mathrm{K}_{\mathrm{U}}$-band (K-lower) and $\mathrm{K}_{\mathrm{A}}$-band (K-above).

The boundaries of the bands are not always followed, and often some overlap is observed. For example, some references consider C-band as 3.7-6.5 GHz and $\mathrm{K}_{\mathrm{u}}$-band as 10.9-12.5 GHz. The bands above $40 \mathrm{GHz}$ have seen several letter designations used, including Q-band, W-band, U-band, and W-band. The ambiguity in letter band designations suggests that they should be used with caution - particularly when the specific frequency is an important consideration. 


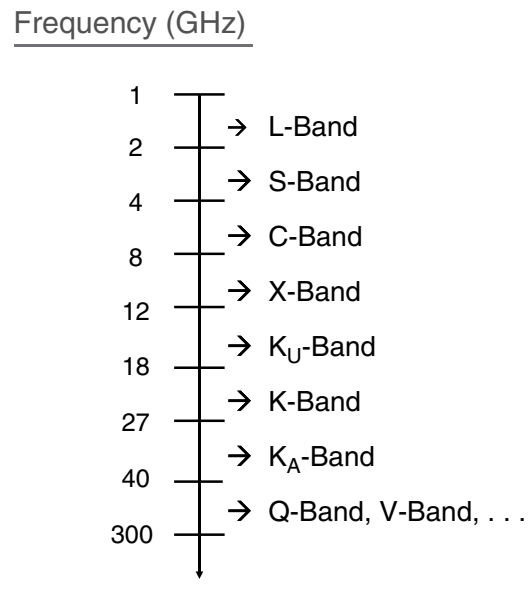

Figure 1.5 Letter band frequency designations

A second designation divides the spectrum from $3 \mathrm{~Hz}$ to $300 \mathrm{GHz}$ into bands based on decade steps of nominal wavelength, as shown in Figure 1.6. This designation is less ambiguous than the letter designation, however, as we shall see in later chapters, most satellite communications links operate within only three or four of the bands, VHF through EHF, with the vast majority of systems in the SHF band.

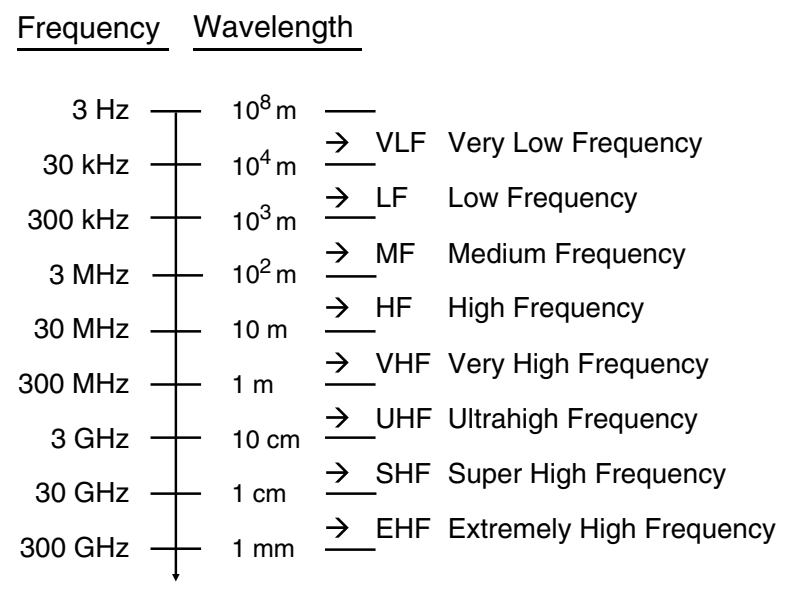

Figure 1.6 Frequency band designations by wavelength

In general, the frequency band designations are useful when general characteristics of satellite communications systems are of interest. When the specific operating carrier frequency or specific frequency band are important, however, the best solution is to specify the frequency directly, rather than using frequency band designations. 


\subsection{Regulatory Process for Satellite Communications}

Satellite operators and owners must operate within constraints of regulations related to fundamental parameters and characteristics of the satellite communications system. The satellite communications system parameters that fall under the regulatory umbrella include:

- choice of radiating frequency;

- maximum allowable radiated power;

- orbit locations (slots) for GSO.

The purpose of the regulation is to minimize radio frequency interference and, to a lesser degree, physical interference between systems. Potential radio interference includes not only other operating satellite systems, but also terrestrial communications systems, and other systems emitting energy in the same frequency bands.

The discipline involved with the development of the technical, analytical, and institutional elements supporting the allocation and regulation of the frequency spectrum is usually referred to as spectrum management or frequency management. Most countries have active organizations, both in the government and the commercial sectors, involved with spectrum management, particularly those organizations responsible for the development of satellite systems or the provision of satellite based services.

There are two levels of regulation and allocation involved in the process: international and domestic. The primary organization responsible for international satellite communications systems regulation and allocation is the International Telecommunications Union (ITU), headquartered in Geneva, Switzerland [15].

The ITU was formed in 1932 from the International Telegraph Union, created in 1865. It is a United Nations Specialized Agency, currently with over 190 members. The ITU structure is similar to the United Nations, with a General Secretariat, elected Administrative Council, boards, and committees for the conduct of Technical and Administrative functions. The ITU has three primary functions:

- allocations and use of the radio-frequency spectrum;

- telecommunications standardization;

- development and expansion of worldwide telecommunications.

The three functions are accomplished through three sectors within the ITU organization: the Radiocommunications Sector (ITU-R), responsible for frequency allocations and use of the radio-frequency spectrum; the Telecommunications Standards Sector (ITU-T), responsible for telecommunications standards; and the Telecommunications Development Sector (ITU-D), responsible for the development and expansion of worldwide telecommunications.

The international regulations developed by the ITU are handed down and processed by each country, where the domestic level regulations are developed. The ITU does not have enforcement powers - each individual country is left to manage and enforce the regulations within its boundaries.

The responsibility for managing regulations in the United States is with the Federal Communications Commission (FCC) and the National Telecommunications and Information Agency (NTIA). Satellite systems operated by the federal government operate through the NTIA, while 
all other systems, including commercial and local government systems, operate through the FCC. The US Department of State coordinates all the frequency and spectrum management activities and represents the US at the ITU and its related organizations. Other countries have their own mechanisms and organizations responsible for the spectrum management function usually government agencies or bureaus working in close cooperation with satellite systems and services providers.

Two attributes determine the specific frequency bands and other regulatory factors for a particular satellite system:

- $\operatorname{service}(s)$ to be provided by the satellite system/network; and

- location $(s)$ of the satellite system/network ground terminals.

Both attributes together determine the frequency band, or bands, where the satellite system may operate.

Figure 1.7 lists the major services as designated by the ITU that are relevant to satellite systems. Some service areas are divided into several sub areas. The mobile satellite service (MSS) area, for example, is further broken down into the aeronautical mobile satellite service (AMSS), the land mobile satellite service (LMSS), and the maritime mobile satellite service (MMSS), depending on the physical locale of the ground based terminals. If the terminals are located on more than one locale, for example on land and sea, then the MSS would apply.

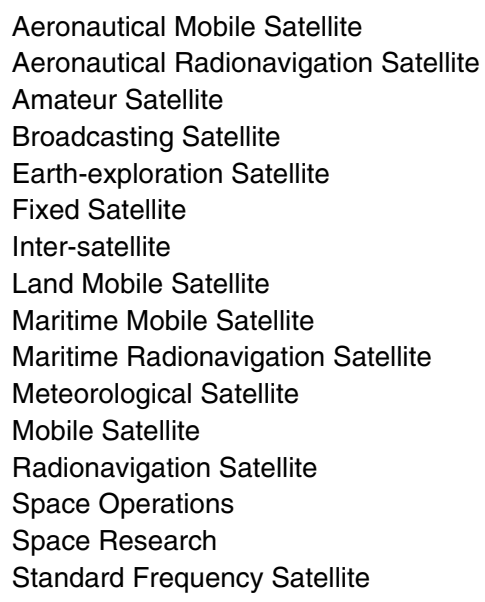

Figure 1.7 Satellite services as designated by the International Telecommunications Union (ITU) (source: ITU [15]; reproduced by permission of International Telecommunications Union)

The second attribute, the location of the earth terminals, is determined by the appropriate service region. The ITU divides the globe into three telecommunications service regions, as shown in Figure 1.8. The three regions divide the earth land areas approximately into the major land masses - Europe and Africa (Region 1), the Americas (Region 2), and the Pacific 


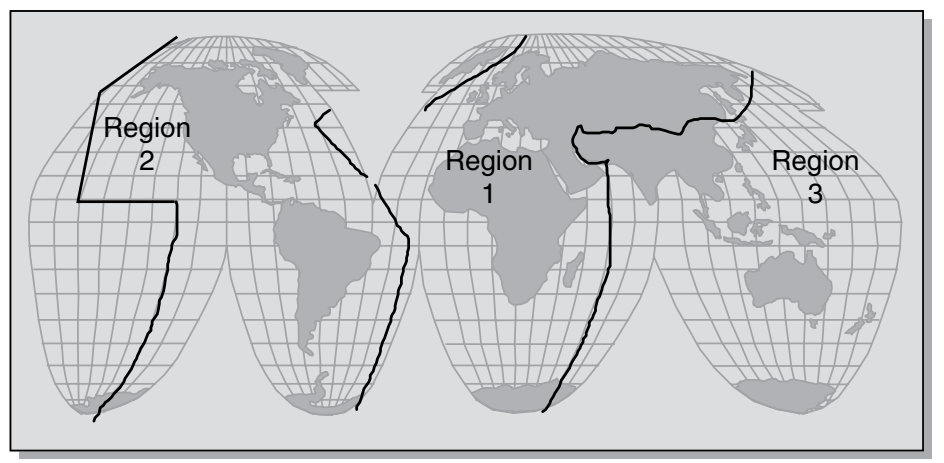

Figure 1.8 ITU telecommunications service regions (source: ITU [15]; reproduced by permission of International Telecommunications Union)

Rim countries (Region 3). Each service region is treated as independent in terms of frequency allocations, because the general assumption is that systems operating in any one of the regions are protected by geographic separation from systems in the other service regions. International frequency allocations are provided for systems operating on a global basis.

\subsection{Overview of Book Structure and Topics}

The material in this book begins with a discussion of some of the basic background disciplines and subsystems inherent in all satellite communications systems. Chapter 2 covers satellite orbits, general orbital mechanics, and focuses on orbits in common use. The parameters required for analysis of the geosynchronous orbit, the most prevalent orbit for current communications satellites, are developed. Chapter 3 introduces the subsystems present in communications satellites, including power, attitude and orbital control, thermal control, and tracking, telemetry, command, and monitoring. The basic elements of the communications satellite payload, the transponder and antenna systems, are introduced.

The next two chapters begin the discussion of the RF link. Transmission fundamentals, system noise, and link performance parameters are developed in Chapter 4. Chapter 5 focuses on link performance for specific types of links, and introduces the concept of percent of time performance specifications for the evaluation of communications satellite systems and networks.

The critical area of transmission impairments introduced to the RF link by the atmosphere is discussed in Chapter 6. Propagation effects on satellite communications are discussed in the context of the frequency band of operation - those operating below about $3 \mathrm{GHz}$, and those operating above $3 \mathrm{GHz}$. Topics covered include ionospheric and tropospheric scintillation, rain attenuation, clouds and fog, and depolarization. Radio noise from a wide range of sources is also covered in detail. Chapter 7 then builds on the discussions of Chapter 6 with a presentation of current modeling and prediction techniques applicable to the evaluation of atmospheric impairments. Most of the models and prediction procedures are presented in a step-by-step solution format, with all the required information in one place, allowing the reader to obtain results directly. Chapter 8 moves to a detailed discussion of modern mitigation techniques 
available to reduce the impact of rain-fading on system performance. Topics discussed include power control, site diversity, orbit diversity, and adaptive coding and modulation.

Chapter 9 puts together all of the pieces from previous chapters to analyze the composite link, which includes the complete end-to-end satellite network. Both frequency translation and on-board processing satellite transponders are included, along with other important subjects such as intermodulation noise and the effects of atmospheric degradations on link performance.

Chapter 10 covers the important topic of satellite multiple access, presented in the context of system performance and the inclusion of critical factors produced on the RF link. Key parameters such as frame efficiency, capacity, and processing gain are developed for the three basic multiple access techniques.

Chapter 11 completes the book with a detailed evaluation of the mobile satellite channel. The unique characteristics of the RF channel environment, and their effects on system design and performance, are highlighted. This chapter is similar in objective and outlook to the composite link evaluation of Chapter 9, but in the context of special considerations unique to the mobile satellite channel.

Appendices are provided for important background material in satellite signal processing elements (for both analog and digital systems), and for mathematical functions used throughout the book.

\section{References}

1 A.C. Clarke, 'Extraterrestrial Relays,' Wireless World, Vol. 51, pp. 305 308, October 1945.

2 M.I. Davis and G.N. Krassner, 'SCORE First Communications Satellite,' Journal of American Rocket Society, Vol. 4, May 1959.

3 L. Jaffe, 'Project Echo Results,' Astronautics, Vol. 6, No. 5, May 1961.

4 E. Imboldi and D. Hershberg, 'Courier Satellite Communications System,' Advances in the Astronautical Sciences, Vol. 8, 1961.

5 Special Issue on Project West Ford, Proceedings of the IEEE, Vol. 52, No. 5, May 1964.

6 K.W. Gatland, Telecommunications Satellites, Prentice Hall, New York, 1964.

7 D.H. Martin, Communications Satellites 1958-1988, The Aerospace Corporation, December 31, 1986.

8 L. Jaffe, 'The NASA Communications Satellite Program Results and Status,' Proceedings of the 15th International Astronautical Congress, Vol. 2: Satellite Systems, 1965.

9 J. Alper and J.N. Pelton, eds., 'The INTELSAT Global System, Progress in Astronautics and Aeronautics,' Vol. 93, AIAA, New York, 1984.

10 J. Almond, 'Commercial Communications Satellite Systems in Canada,' IEEE Communications Magazine, Vol. 19, No. 1, January 1981.

11 W.N. Redisch and R.L. Hall, 'ATS 6 Spacecraft Design/Performance,' EASCON'74 Conference Record, October 1974.

12 Special Issue on ATS 6, IEEE Transactions on Aerospace and Electronics Systems, Vol. 11, No. 6, November 1975.

13 D.L. Wright and J.W.B. Day, 'The Communications Technology Satellite and the Associated Ground Terminals for Experiments,' AIAA Conference on Communications Satellites for Health/Education Applications, July 1975.

14 L.J. Ippolito, 'Characterization of the CTS 12 and $14 \mathrm{GHz}$ Communications Links Preliminary Measurements and Evaluation,' International Conference on Communications: ICC'76, June 1976.

15 International Telecommunications Union, www.itu.int. 
